\title{
Ultrafast Population Dynamics of Surface-Active Dyes during Electrochemically Controlled Ion Transfer across a LiquidlLiquid Interface
}

\author{
Astrid J. Olaya, Pierre-François Brevet, Evgeny A. Smirnov, and Hubert H. Girault* \\ Laboratoire d'Electrochimie Physique et Analytique, Station 6, Ecole Polytechnique Fédérale de Lausanne, CH-1015 Lausanne, \\ Switzerland
}

ABSTRACT: We present for the first time experimental measurements on the ultrafast population dynamics of the model surface-active dye methyl orange (MO) during its ion transfer across the waterl1,2-dichloroethane interface by using time-resolved surface second harmonic generation (TR-SSHG). The interfacial dynamics reveals that the population of $\mathrm{MO}$ relaxes to the ground state within $160 \mathrm{fs}$ without any significant contribution of the molecular reorientation. It is concluded that the MO transfer across the interface considerably affects the population of $\mathrm{MO}$ at the interface but has no effect on the dynamics of the molecule itself.

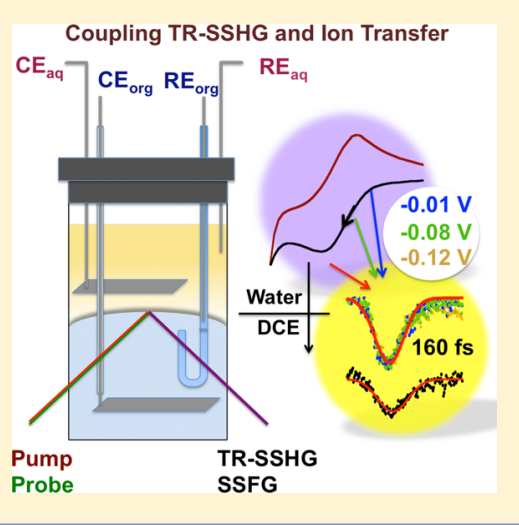

\section{INTRODUCTION}

Liquid-liquid interfaces are ubiquitous in nature and the siege of vital processes for living organisms. Ion transfer across cellular channels or solvent extraction processes are fields of research where the study of the kinetics and thermodynamics of charge transfer processes at the interface between two immiscible electrolyte solutions (ITIES) is mandatory. ITIES indeed provide the possibility to study various transfer processes such as ion transfer, assisted ion transfer, or heterogeneous electron transfer. They are generally considered as bioinspired systems and catalytic platforms suitable for the study of key reactions involving charge transfer processes in biocatalysis, respiration, ion pumping, photosynthesis, or selfassembly, to name just a few. ${ }^{1-10}$ The efficiency at which interfacial processes and reactions are carried out across ITIES strongly depends on the structure and the dynamics of the interfacial region, including electrostatic and intermolecular forces. Therefore, the implementation of interfacial characterization techniques selective to the interface is extremely important in order to get reliable insights into the interfacial structure and the reaction mechanisms. ${ }^{1-16}$ In this regard, fluorescence and Raman microspectroscopy have been used to analyze ion association and interfacial complexation. However, the spatial resolution of these techniques still depends on the light diffraction limit unless cumbersome near-field optical methods are introduced. Thus, over the recent years, inherently surface specific nonlinear quadratic nonlinear optical techniques like surface second-harmonic generation (SSHG) and sum frequency generation (SSFG) have been introduced. The surface specificity of these nonlinear optical methods stems from the vanishing of the second-order nonlinear susceptibility in centrosymmetric media within the electric dipole approx- imation. In particular, SSHG has been successfully implemented to study adsorption of anionic surfactants and dyes like methyl orange (MO) and porphyrins at liquid interfaces. Furthermore, the ion transfer properties of $\mathrm{MO}$ and porphyrin self-assemblies have been also addressed by SSHG as a function of external electrical polarization. ${ }^{11-16}$

To unravel a more complete picture of the ion transfer process, the dynamic behavior of the adsorbed species during ion transfer remains to be investigated. To this purpose, namely to follow the dynamics of photoactive molecules on short time scales, time-resolved (TR) techniques must be implemented. For instance, TR total internal reflection fluorescence (TRTIRF) has provided valuable information on the aggregation and the reorientational dynamics of Coumarin 343 for instance. However, this technique still suffers from a limited time resolution (about 20 ps) and the lack of true sensitivity to the interface, intrinsically limited by its evanescent wave penetration depth. Therefore, second-order nonlinear optical techniques like TR-SSHG and vibrational or electronic TRSSFG have been successfully implemented to study dynamic processes at interfaces. ${ }^{10,17,18}$ In particular, TR-SSHG at a single wavelength has been used to study the solvent dynamics of Coumarin 314 at the waterlair interface. ${ }^{19,20}$ Also, the study of the reorientional dynamics of adsorbed dyes gives important information on the local environment of a molecule. However, such studies are rather complicated at interfaces because the generation of the optical signals also depends on the intrinsic quadratic nonlinearity of the molecules, their surface coverage,

Received: July 28, 2014

Revised: October 6, 2014

Published: October 8, 2014 
and potentially to their interactions. Nevertheless, by using TRSSHG, it has been found that unlike bulk solutions, the reorentational dynamics at interfaces is developed through two different motions, namely the in-plane and the out-of-plane motions. ${ }^{19,21-23}$ TR-SSHG studies on malachite green (MG) at airlwater and alkanelwater interfaces also showed how this technique could be successfully implemented to study the relative affinity of ions for interfaces. ${ }^{10,18,24,25}$

Many important photochemical reactions occurring in nature take place across polarized interfaces, like the oxidation of water in the photosynthetic apparatus for example. The study of the effect of charge transfer on the population dynamics of surface active molecules is thus of interest. We report such a study here where the ultrafast dynamics of a model surface-active organic dye is monitored during its ion transfer across the waterl1,2dichloreoethane interface under controlled electrical polarization.

\section{EXPERIMENTAL SECTION}

Chemicals. All solvents and chemicals were used as received without further purification. Sodium Methyl Orange (NaMO, $95 \%)$ was purchased from Aldrich. Bis(triphenylphosphoranylidine)ammonium chloride (BACl, 98\%), tetrakis(pentafluorophenyl)borate ethyl etherate (LiTB purum), and lithium chloride ( $\mathrm{LiCl}>99 \%)$ were purchased from Fluka. 1,2-Dicholoroethane (DCE, 99+\% for spectroscopy, laser grade) was purchased from ACROS. Bis(triphenylphosphoranylidine) ammonium tetrakis(pentafluorophenyl)borate (BATB) was synthesized by metathesis of a 1:1 mixture of $\mathrm{BACl}$ and $\mathrm{LiTB}$, respectively, dissolved in methanol-water mixtures $(\mathrm{v}: \mathrm{v}=2: 1)$, followed by recrystallization in acetone. All the solutions were prepared in Milli-Q grade water.

Optical Measurements. Scheme 1 illustrates the collinear TR-SSHG setup. The fundamental beam delivered by a

Scheme 1. Electrochemical TR-SSHG Setup Schematics ${ }^{a}$

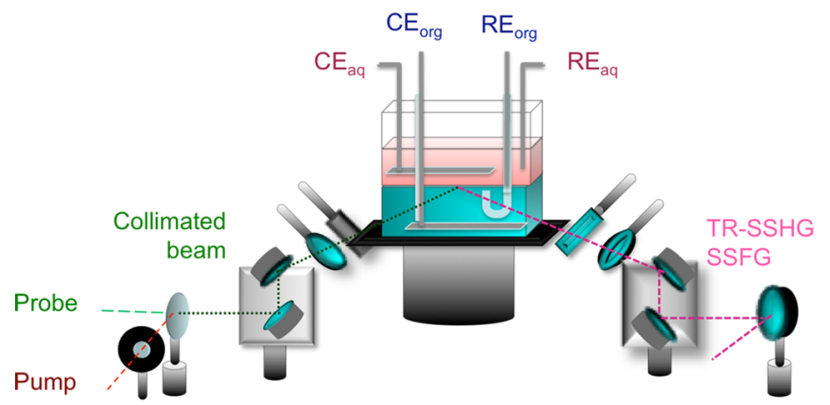

${ }^{a} \mathrm{CE}=$ counter electrode, $\mathrm{RE}=$ reference electrode, $\mathrm{Aq}=$ aqueous phase, and Org = organic phase.

Ti:sapphire amplified femtosecond laser (LIBRA USP HE, Coherent) providing pulses with a central wavelength of 800 $\mathrm{nm}$ and energy of $4.5 \mathrm{~mJ}$ was used as the pump beam. Part of this fundamental beam was used to pump an OPA (OPerA SOLO system, Light Conversion) in order to obtain wavelength tunable pulses. The OPA was pumped with $1.2 \mathrm{~mJ}$ pulses of the fundamental beam. The length of the pump arm was computer controlled for time synchronization with the probe beam, and a power control system (a half-wave plate coupled to a polarizer) was used to adjust the energy of the pump down to $4.1 \mu \mathrm{J}$ and below. A motorized rotating half- have plate was used to linearly set the angle of polarization. The path length of the probe arm was kept fixed because of the sensitivity of SSHG to misalignment, and the energy of the probe arm was also controlled, typically below $30 \mu \mathrm{J}$. Unlike classical TR-SSHG setups, the present configuration was collinear, thereby facilitating the alignment and synchronization procedure. In order to remove any SHG radiation prior to the cell, three low-pass filters (Melles-Griot, model RG715) were placed on the collinear beam path. Then, a converging lens with a focal length of $+100 \mathrm{~mm}$ was focusing the beams onto the waterIDCE interface from the organic phase. Total internal reflection (TIR) was achieved with an angle of incidence of about $70^{\circ}$ for both beams. The reflected SHG radiation was then separated from the fundamental beam with a high pass filter (Melles-Griot, model BG38), and the collected SHG signals were collimated with a converging lens with focal length of $+100 \mathrm{~mm}$ and filtered with successive filters (Melles-Griot, model BG38) before entering the monochromator (JobinYvon, model HR320). Finally, the SHG photons were detected with a photomultiplier tube (Hamamatsu, model R928). A power meter (Ophir, model PE-10) was used to continuously monitor the fundamental beam energy, and the SHG signal was averaged over many laser shots. The resulting SHG spectra were all corrected for background noise. In order to find the origin of time at $t=0$, the pump beam was delayed with respect to the probe until the generation of sum frequency was observed between the pump and the probe beams. Posteriorly, the power of the pump was increased in order to promote excitation of the interfacial molecules reflected by a substantial decrease of the SSH intensity, and the pump was further delayed in order to construct the TR-SSHG profiles. In order to avoid problems related to any physical perturbation of the interface other than the optical effects (white light generation, degradation of the interface, evaporation of the organic phase, or liquid bubbles formation), the power of the pump and the probe beams was attenuated below 4.1 and $0.5 \mu \mathrm{J}$, respectively. SSFG from the pump and probe beams from the interface was used for the determination of the time resolution of the experimental setup. The instrument time resolution was found to be $115 \pm 0.8 \mathrm{fs}$.

Ion-Transfer Voltammetry Coupled to TRSSHG. Iontransfer voltammetry measurements at the water|DCE interface were performed in a four-electrode cell following the configuration described previously by Nagatani et al. ${ }^{26}$ (see Scheme 1). A commercial potentiostat (IVIUM) was used. In the four-electrode cell, two reference electrodes polarize the interface and two platinum counter electrodes provide the current. The external potential was applied by means of two silver/silver chloride $(\mathrm{Ag} / \mathrm{AgCl})$ reference electrodes connected to the aqueous and DCE phases, respectively, by means of Luggin capillaries. ${ }^{3}$ The Galvani potential difference $\Delta_{\mathrm{o}}^{\mathrm{w}} \phi$ was estimated by taking the formal ion transfer potential of $\mathrm{TMA}^{+}$ as $0.18 \mathrm{~V} .{ }^{9}$ The schematic representation of the initial composition of the four-electrode cell is illustrated in Scheme 2.

In order to analyze the static SSHG response of $\mathrm{MO}$ at the interface, the SSH intensity was recorded as a function of the applied potential. Subsequently, the potential was fixed to three different values while recording the TR-SSHG profiles of MO as described before in order to study the effect of charge transfer on the dynamics of MO. These experiments were performed with the probe p-polarized and the pump spolarized. 
Scheme 2. Initial Electrochemical Cell Composition of the Four-Electrode Cell Used To Study the Transfer of MO

$$
\mathrm{Ag}|\mathrm{AgCl}| \begin{gathered}
100 \mu \mathrm{M} \mathrm{MO} \\
10 \mathrm{mM} \mathrm{LiCl} \\
\text { Water }
\end{gathered}\left|\begin{array}{c}
5 \mathrm{mM} \text { BATB } \\
\text { DCE }
\end{array}\right| \begin{gathered}
10 \mathrm{mM} \mathrm{BACl} \\
10 \mathrm{~m} \mathrm{LiCl}
\end{gathered}|\mathrm{AgCl}| \mathrm{Ag}
$$

\section{RESULTS AND DISCUSSION}

In a previous work it was determined that the SSHG spectrum of MO is made by two bands centered at 430 and $470 \mathrm{~nm}$ and respectively attributed to the $\pi-\pi^{*}$ transitions of the nonhydrated $\left[\mathrm{MO}^{-}\right]$and the hydrated $\left[\mathrm{MO}^{-} / \mathrm{H}_{2} \mathrm{O}\right]$ forms of $\mathrm{MO}^{27}$ (see Figure 1). In this work we focused on the hydrated

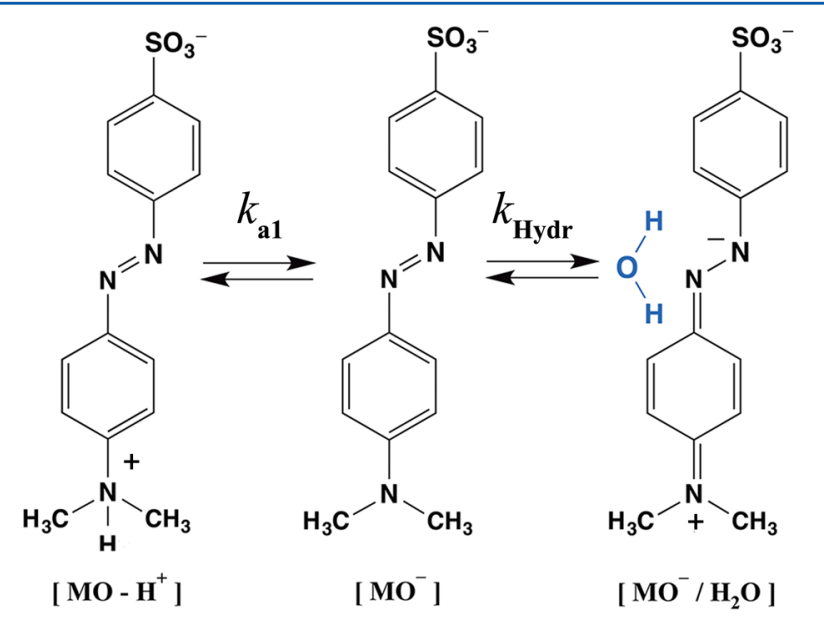

Figure 1. Chemical structures of the stable MO moieties in bulk solutions.

$\left[\mathrm{MO}^{-} / \mathrm{H}_{2} \mathrm{O}\right]$ form since it provided the most intense SSHG response. However, considering that the $470 \mathrm{~nm}$ SSHG band is rather broad, we recorded the time profiles at $460 \mathrm{~nm}$ instead, in order to get a better compromise between optics transmission and OPA power.

Considering the $\mathrm{pH}$ of 6.5 of the MO solutions used in these experiments and the $\mathrm{p} K_{\mathrm{a}}$ of 3.4 of $\mathrm{MO}$, both the nonhydrated and hydrated $\mathrm{MO}$ forms must be in their anionic form ${ }^{27}$ (see Figure 1). It has been also established that the interfacial equilibrium between the two known forms of $\mathrm{MO}$ in water is identical to the equilibrium occurring in the bulk of the aqueous phase and that both forms sit principally on the aqueous side of the interface. ${ }^{27}$

TR-SSHG relaxation profiles of $\left[\mathrm{MO}^{-} / \mathrm{H}_{2} \mathrm{O}\right]$ were recorded upon two-photon excitation at $800 \mathrm{~nm}$ by using the setup illustrated in Scheme 1 and fully described in the Experimental Section. Interfacial molecules were selectively excited at $70^{\circ}$ incidence (coming from the bottom phase) with respect to the liquid $\rightarrow$ liquid interface with linearly polarized pump and probe pulses, generating an SSHG signal from both beams simultaneously and therefore, as a result, a surface sum frequency generation (SSFG) signal. The SSFG response was in turn used to determine the time resolution of the instrument $(115 \pm 0.8 \mathrm{fs})$; see inset in Figure 2. The depletion of the SSHG signal displayed in the relaxation profiles shown in Figure 2 is attributed to the partial bleaching of the ground state population of the MO species. Large contrasts were obtained due to the use of high pump powers and the large MO

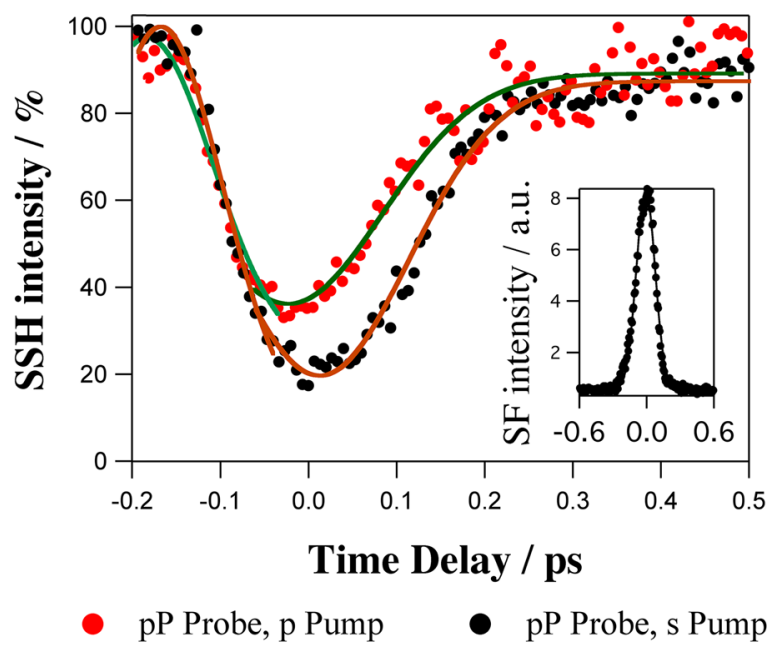

Figure 2. TR-SSHG profiles recorded under two-photon excitation $(800 \mathrm{~nm})$ with different pump-probe polarization configurations. The time resolution of the experiment was estimated by SFG setting the wavelengths of pump and probe at 800 and $920 \mathrm{~nm}$, respectively (inset). The curves are normalized with respect to the maximum SSHG intensity observed for the ground species.

absorption cross section. Nevertheless, care was taken to prevent the appearance of any spurious nonlinear effects. Subsequently, the repopulation of the ground state accounts for the recovery of the SSHG signal. The relaxation profiles were adjusted by using an asymmetric Gaussian-like function to account for the different rising and decaying times. The asymmetry of the profiles indicates that the population relaxation of the MO species is longer than the instrument time resolution of $115 \pm 5 \mathrm{fs}$. A time constant of $160 \pm 10 \mathrm{fs}$ corresponding to the ground state population recovery time was determined regardless of the polarization of the pump and probe beams. Differences were observed only in the depletion ratio with respect to the SSHG intensity for the ground state. The absence of dependence with the polarization of the probe beam indicates that no orientational motion occur, as expected for such a fast relaxation time. Also, when the pump was spolarized, the contrast was about $83 \%$ (see Figure 2), whereas it was only about $65 \%$ for the pump p-polarized irrespective of the polarization of the probe. In order to explain such small difference between the two configurations, the orientation of $\mathrm{MO}$ at the interface must be taken into account.

In 2005, Del Nero et al. ${ }^{28}$ estimated the optimized geometries of the azonium and alkaline forms of $\mathrm{MO}$ in solution by a combination of semiempirical and $a b$ initio methods in order to calculate their second hyperpolarizabilities. In both alkaline and acidic media, the trans conformation was found to be dominant and essentially planar. When comparing both the trans and the cis conformations, the trans was found to have a larger dipolar moment and to be more stable than the cis one. Therefore, the trans conformation was assumed to dominate the nonlinear response due to a larger first hyperpolarizability. Considering that the transition dipole moment of the trans configuration is essentially oriented along the main molecular axis, we also assumed that in the absence ofthe pump beam the angle of orientation of the transition moment of $\mathrm{MO}$ with respect to the interface is similar to that estimated by Rinuy et al., ${ }^{27}$ namely $43 \pm 2^{\circ}$ for $\left[\mathrm{MO}^{-} / \mathrm{H}_{2} \mathrm{O}\right]$ and $34 \pm 2^{\circ}$ for $\left[\mathrm{MO}^{-}\right]$. Considering such an intermediate angle, it is not surprising that the difference in 
depletion of the SSHG signal with the polarization of the pump beam did not exceed $20 \%$.

In order to study the dynamics of MO during the ion transfer across the interface, an external electrical field was applied across the interface by using a four-electrode cell described in the Experimental Section with the chemical composition illustrated in Scheme 2.

Figure 3 displays the voltammetric response associated with the transfer of MO from the aqueous to the organic phase. By
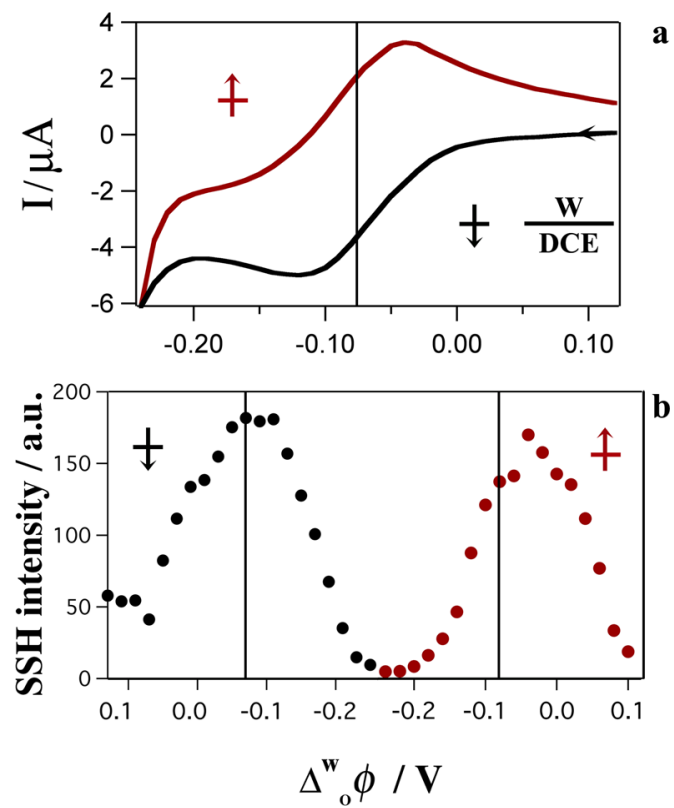

Figure 3. (a) Ion-transfer voltammogram of $100 \mu \mathrm{M}$ MO $(20 \mathrm{mV}$ $\mathrm{s}^{-1}$ ); the inset arrows indicate the direction of the ion transfer. (b) SSHG intensity of $100 \mu \mathrm{M}$ MO versus electrical potential recorded under electrical polarization of the interface by using a four-electrode system. In order to compare with Figure 2, the curves are normalized with respect to the maximum SSHG intensity obtained for the ground species in the absence of electrical polarization of the interface.

convention, positive currents are attributed to the transfer of a positive charge from the aqueous to the organic phase or to the transfer of a negative charge from the organic to the aqueous phase. Taking into account the standard transfer potential of the ions constituting the supporting electrolytes in both phases, the potential window is limited by the transfer of $\mathrm{Cl}^{-}$at negative potentials and by $\mathrm{Li}^{+}$at positive potentials. The standard transfer potential, $\Delta_{\mathrm{o}}^{\mathrm{w}} \phi_{1 / 2}$, of $\mathrm{MO}$ was found to be $-0.082 \mathrm{~V}$ at $20 \mathrm{mV} \mathrm{s}^{-1}$, in agreement with previous results. ${ }^{29}$ The MO system was analyzed first in the ground state by recording the SSHG response under electrical polarization and in the absence of pump beam (Figure $3 b$ ), scanning the applied potential from -0.2 to $0.2 \mathrm{~V}$ forward and backward as it is normally done when recording a cyclic voltammogram. The plot of the SSHG intensity versus the electrical potential shows that the SSHG signal gradually increases as the applied potential approaches $\Delta_{\mathrm{o}}^{\mathrm{w}} \phi_{1 / 2}$. In line with a previous static study, this SSHG intensity increase is attributed to the accumulation of the MO molecules to the interface and the decrease to the depletion of the molecules away from the interface owing to the transfer as the potential is swept toward negative values. ${ }^{29}$ The maximum of the SSHG intensity for the forward peak is higher than that obtained for the backward peak owing to the light absorption in the organic phase after irreversible ion-transfer of part of the MO molecules.

In order to study the effect of charge transfer on the dynamics of $\mathrm{MO}$, relaxation profiles were recorded upon electrical polarization by fixing the potential to three different values while recording the TR-SSHG signal. These experiments were performed with the probe p-polarized and the pump spolarized. Figure 4 shows the dynamics of the molecules

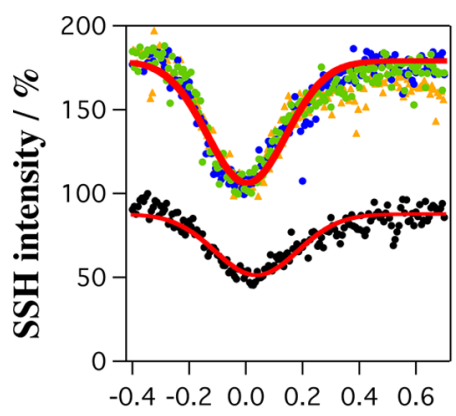

$$
\begin{aligned}
& \text { Time Delay /ps } \\
& \begin{array}{ll}
\text { - Without Polarization } & -0.01 \mathrm{~V} \\
-0.08 \mathrm{~V} & \Delta-0.12 \mathrm{~V}
\end{array}
\end{aligned}
$$

Figure 4. TR-SSHG profiles recorded under two-photon excitation $(800 \mathrm{~nm})$ for the resonance at $460 \mathrm{~nm}$ with and without electrical polarization of the interface. The probe and the pump being $\mathrm{pP}$ and $\mathrm{s}$ polarized, respectively. In order to compare with Figure 2, the curves are normalized with respect to the maximum SSH intensity obtained for the ground species in the absence of electrical polarization of the interface.

adsorbed at the interface, with and without polarization. The curves are normalized with respect to the maximum SSHG intensity recorded in the absence of electric polarization. This analysis shows that the dynamics of $\mathrm{MO}$ is independent of the applied potential, effectively unaffected by the electrical polarization of the interface. Hence, in the case of the MO ion transfer, it is suggested that the population relaxation is far too fast to be affected by the applied potential. The overall population however follows the applied potential dependence observed during the static experiment. Besides, the depletion ratio is similar to the one reported in the absence of pump excitation, indicating that the ion transfer does not alter the population equilibrium between the excited and ground state MO molecules. These results suggest that the transfer properties of the two excited and ground state MO species are similar. The fast dynamics observed here likely occurs on a time scale too fast for any transfer to take place.

\section{CONCLUSION}

In this experimental study, the dynamics of the surface-active dye MO is monitored during its ion transfer process across the waterIDCE interface. The dynamics reveals that the MO species relax to the ground state within $160 \pm 10 \mathrm{fs}$ after two-photon excitation with a pump beam set at $800 \mathrm{~nm}$ and that the relaxation time remains unaffected by the electrical polarization of the interface. From the light polarization analysis, it appears that there is no time for any orientational processes to occur.

The ion transfer of MO across the waterIDCE interface is thus investigated for both the excited and ground state molecules. The fast population relaxation prevents the 
observation of a difference between the excited and ground state MO ion transfer properties and therefore to discuss in light of experimental results the transfer properties difference between the ground and excited species.

\section{AUTHOR INFORMATION}

\section{Corresponding Author}

*E-mail: hubert.girault@epfl.ch (H.H.G.).

\section{Present Address}

P.-F.B.: Institut Lumière Matière, UMR CNRS 5306, Université Claude Bernard Lyon 1, 43 Boulevard du 11 Novembre 1918, F-69622 Villeurbanne cedex, France.

\section{Notes}

The authors declare no competing financial interest.

\section{ACKNOWLEDGMENTS}

This research was supported by the National Center in Competence in Research Molecular Ultrafast Science and Technology (NCCR MUST) funded by the Swiss National Science Foundation and the Ecole Polytechnique Fédérale de Lausanne (Switzerland).

\section{REFERENCES}

(1) Li, F.; Su, B.; Salazar, F. C.; Nia, R. P.; Girault, H. H. Detection of Hydrogen Peroxide Produced at a Liquid/Liquid Interface Using Scanning Electrochemical Microscopy. Electrochem. Commun. 2009, 11, 473-476.

(2) Partovi-Nia, R.; Su, B.; Li, F.; Gros, C. P.; Barbe, J. M.; Samec, Z.; Girault, H. H. Proton Pump for $\mathrm{O}_{2}$ Reduction Catalyzed by 5,10,15,20-Tetraphenylporphyrinatocobalt(II). Chem.-Eur. J. 2009, $15,2335-2340$.

(3) Hatay, I.; Su, B.; Li, F.; Mendez, M. A.; Khoury, T.; Gros, C. P.; Barbe, J. M.; Ersoz, M.; Samec, Z.; Girault, H. H. Proton-Coupled Oxygen Reduction at Liquid-Liquid Interfaces Catalyzed by Cobalt Porphine. J. Am. Chem. Soc. 2009, 131, 13453-13459.

(4) Su, B.; Hatay, I.; Li, F.; Partovi-Nia, R.; Méndez, M. A.; Samec, Z.; Ersoz, M.; Girault, H. H. Oxygen Reduction by Decamethylferrocene at Liquid/Liquid Interfaces Catalyzed by Dodecylaniline. J. Electroanal. Chem. 2010, 639, 102-108.

(5) Su, B.; Hatay, I.; Trojanek, A.; Samec, Z.; Khoury, T.; Gros, C. P.; Barbe, J. M.; Daina, A.; Carrupt, P. A.; Girault, H. H. Molecular Electrocatalysis for Oxygen Reduction by Cobalt Porphyrins Adsorbed at Liquid/Liquid Interfaces. J. Am. Chem. Soc. 2010, 132, 2655-2662.

(6) Mendez, M.; Partovi-Nia, R.; Ge, P.; Olaya, A.; Hojeij, M.; Girault, H. H. Molecular Electrocatalysis at Soft Interfaces. Phys. Chem. Chem. Phys. 2010, 12, 15163-15171.

(7) Olaya, A. J.; Schaming, D.; Brevet, P. F.; Nagatani, H.; Zimmermann, T.; Vanicek, J.; Xu, H.-J.; Gros, C. P.; Barbe, J. M.; Girault, H. H. Self-Assembled Molecular Rafts at LiquidlLiquid Interfaces for Four-Electron Oxygen Reduction. J. Am. Chem. Soc. 2012, 134, 498-506.

(8) Olaya, A. J.; Schaming, D.; Brevet, P.-F.; Nagatani, H.; Xu, H.-J.; Meyer, M.; Girault, H. H. Interfacial Self-Assembly of Water-Soluble Cationic Porphyrins for the Reduction of Oxygen to Water. Angew. Chem., Int. Ed. 2012, 51, 6447-6451.

(9) Olaya, A. J.; Méndez, M. A.; Cortés-Salazar, F.; Girault, H. H. Voltammetric Determination of Extreme Standard Gibbs Ion Transfer Energy. J. Electroanal. Chem. 2010, 644, 60-66.

(10) Fita, P.; Punzi, A.; Vauthey, E. Local Viscosity of Binary Water + Glycerol Mixtures at Liquid/Liquid Interfaces Probed by TimeResolved Surface Second Harmonic Generation. J. Phys. Chem. C 2009, 113, 20705-20712.

(11) Fujiwara, K.; Monjushiro, H.; Watarai, H. Non-Linear Optical Activity of Porphyrin Aggregate at the Liquid/Liquid Interface. Chem. Phys. Lett. 2004, 394, 349-353.
(12) Fujiwara, K.; Monjushiro, H.; Watarai, H. Total Internal Reflection Second-Harmonic Generation Spectrometer System Optimized for the Liquid/Liquid Interface. Rev. Sci. Instrum. 2005, 76, 023111-1-023111-5.

(13) Fujiwara, K.; Wada, S.; Monjushiro, H.; Watarai, H. IonAssociation Aggregation of an Anionic Porphyrin at the Liquid/Liquid Interface Studied by Second Harmonic Generation Spectroscopy. Langmuir 2006, 22, 2482-2486.

(14) Conboy, J. C.; Richmond, G. L. Total Internal Reflection Second Harmonic Generation from the Interface Between Two Immiscible Electrolyte Solutions. Electrochem. Acta 1995, 40, 28812886.

(15) Nagatani, H.; Piron, A.; Brevet, P. F.; Fermin, D. J.; Girault, H. H. Surface Second Harmonic Generation of Cationic Water-Soluble Porphyrins at the Polarized Waterl1,2-Dichloroethane Interface. Langmuir 2002, 18, 6647-6652.

(16) Eugster, N.; Sreenivasan, K. P.; Su, B.; Girault, H. H. 2Dimensional Porphyrin Self-Assemblies at Molecular Interfaces. Langmuir 2006, 22, 1112-1120.

(17) Eisenthal, K. B. Liquid Interfaces Probed by Second-Harmonic and Sum-Frequency Spectroscopy. Chem. Rev. 1996, 96, 1343-1360.

(18) Fedoseeva, M.; Fita, P.; Punzi, A.; Vauthey, E. Salt Effect on the Formation of Dye Aggregates at Liquid/Liquid Interfaces Studied by Time-Resolved Surface Second Harmonic Generation. J. Phys. Chem. C 2010, 114, 13774-13781.

(19) Zimdars, D.; Dadap, J. I.; Eisenthal, K. B.; Heinz, T. F. Anisotropic Orientational Motion of Molecular Adsorbates at the AirWater Interface. J. Phys. Chem. B 1999, 103, 3425-3433.

(20) Zimdars, D.; Dadap, J. I.; Eisenthal, K. B.; Heinz, T. F. Femtosecond Dynamics of Solvation at the Air/Water Interface. Chem. Phys. Lett. 1999, 301, 112-120.

(21) Nguyen, K. T.; Shang, X.; Eisenthal, K. B. Molecular Rotation at Negatively Charged Surfactant/Aqueous Interfaces. J. Phys. Chem. B 2006, 110, 19788-19792.

(22) Shang, X.; Nguyen, K.; Rao, Y.; Eisenthal, K. B. In-Plane Molecular Rotational Dynamics at a Negatively Charged Surfactant/ Aqueous Interface. J. Phys. Chem. C 2008, 112, 20375-20381.

(23) Gengeliczki, Z.; Rosenfeld, D. E.; Fayer, M. D. Theory of Interfacial Orientational Relaxation Spectroscopic Observables. J. Chem. Phys. 2010, 132, 244703-1-244703-19.

(24) Punzi, A.; Martin-Gassin, G.; Grilj, J.; Vauthey, E. Effect of Salt on the Excited-State Dynamics of Malachite Green in Bulk Aqueous Solutions and at Air/Water Interfaces: A Femtosecond Transient Absorption and Surface Second Harmonic Generation Study. J. Phys. Chem. C 2009, 113, 11822-11829.

(25) Richert, S.; Fedoseeva, M.; Vauthey, E. Ultrafast Photoinduced Dynamics at Air/Liquid and Liquid/Liquid Interfaces. J. Phys. Chem. Lett. 2012, 3, 1635-1642.

(26) Nagatani, H.; Samec, Z.; Brevet, P.-F.; Fermin, D. J.; Girault, H. $\mathrm{H}$. Adsorption and Aggregation of meso-Tetrakis(4-Carboxyphenyl)porphyrinato Zinc(II) at the Polarized Waterl1,2-Dichloroethane Interface. J. Phys. Chem. B 2002, 107, 786-790.

(27) Rinuy, J.; Piron, A.; Brevet, P. F.; Blanchard-Desce, M.; Girault, H. H. Intramolecular Electron Density Redistribution upon Hydrogen Bond Formation in the anion Methyl Orange at the Water/1,2Dichloroethane Interface Probed by Phase Interference Second Harmonic Generation. Chem.-Eur. J. 2000, 6, 3434-3441.

(28) Del Nero, J.; De Araujo, R. E.; Gomes, A. S. L.; De Melo, C. P. Theoretical and Experimental Investigation of the Second Hyperpolarizabilities of Methyl Orange. J. Chem. Phys. 2005, 122, 104506$1-104506-6$.

(29) Piron, A.; Brevet, P. F.; Girault, H. H. Surface Second Harmonic Generation Monitoring of the Anion Methyl Orange During Ion Transfer Reactions across a Polarized Water. J. Electroanal. Chem. 2000, 483, 29-36. 